\title{
Parsec Scale Properties of Brightest Cluster Galaxies.
}

\author{
Elisabetta Liuzzo* ${ }^{* 1,2}$, Gabriele Giovannini ${ }^{1,2}$ and Marcello Giroletti ${ }^{1}$ \\ ${ }^{1}$ Istituto di Radioastronomia, INAF, via Gobetti 101, 40129 Bologna. \\ ${ }^{2}$ Dipartimento di Astronomia, Universita' di Bologna,via Ranzani 1, 40127 Bologna, Italy \\ E-mail: liuzzoeira.inaf.it. ggiovann@ira.inaf.it. \\ girolettieira.inaf.it
}

We present new VLBI observations of a complete sample of Brigthest Cluster Galaxies (BCGs) in nearby Abell Clusters. These data show a possible difference between BCGs in cool core clusters (two-sided parsec scale jets) and in non cool core clusters (one-sided parsec scale jet). We suggest that this difference could be due to the jet interaction with the surrounding medium. More data are necessary to discuss whether parsec-scale properties of BCGs are influenced by their peculiar morphology and position at the center of rich galaxy clusters.

The 9th European VLBI Network Symposium on The role of VLBI in the Golden Age for Radio Astronomy and EVN Users Meeting

September 23-26, 2008

Bologna, Italy

\footnotetext{
*Speaker.
} 


\section{Introduction}

Brightest Cluster Galaxies (BCGs) are a unique class of objects [11]. These galaxies are the most luminous and massive galaxies in the Universe. Most of them are cD galaxies with extended envelopes of excess light, but they can be also giant $\mathrm{E}$ and $\mathrm{D}$ galaxies. The optical morphology often shows evidence of past or recent galaxy mergers (e.g. multiple nuclei). Moreover, they tend to lie very close to the peak of the cluster X-ray emission and in the velocity space they sit near the cluster rest frame. All these properties indicate that they could have a quite unusual formation history compared to other E galaxies.

In the radio band, BCGs are more likely to be radio-loud than other galaxies of the same mass [1]. Some BCGs have a standard tailed structure, either extended on the kpc scale (e.g. 3C465 in A2634), or with a small size (e.g. NGC4874 in Coma cluster). In other cases, they show a diffuse and amorphous radio morphology that is rare in the general radio population, but very often present in BCGs in cooling core clusters of galaxies. The presence of X-ray cavities in the emitting gas coincident with the presence of radio lobes can also be the proof of the interplay between the radio activity of BCGs and the arrest or slow down of the cooling process at the cluster centers [2,3]. This scenario is in agreement with the recent result that in every/most of cooling core clusters is present an active radio BCG [5].

However, a few points need a deeper study: not all BCGs are strong radio sources and a restarting activity with a not too long duty cycle is necessary to justify the slow down of cool ing processes. Moreover, it is not clear if radio properties of BCGs in cooling flow clusters are systematically different from those of BCGs in merger clusters; we note that in cooling clusters the kpc scale radio morphology of BCGs is diffuse and relaxed and it is often surrounded by an extended low-brightness radio emission that takes the form of mini-halo [7]. However, extended 'normal' sources have been also found (e.g. Hydra A, [9]). In merging clusters the most common morphology is a Wide Angle Tail source but point-like as well as core-halo sources are also present.

On the parsec scale, BCGs are not yet well studied as a class of sources. Only a few of them have been observed, being well known radio galaxies. In some cases, they look like normal FRI radio galaxies with relativistic collimated jets. Jets are often one-sided because of Doppler boosting effects (e.g. 3C465 in A2634 and 0836+29 in A690 [10]), although there are also cases where twosided symmetric jets are present in VLBI images, and the presence of highly relativistic jets is not certain (e.g. 3C338 in A2199 [4], and Hydra A in A780 [9]).

\section{Observations and Results.}

To study parsec scale properties of BCGs we selected a complete sample of BCGs in nearby Abell clusters with the following constraints: 1) Distance Class $\leq 2$, and 2) Declination $>0^{\circ}$. All clusters have been included without constraints on cluster conditions (e.g cooling) and no selection is present on the $\mathrm{BCG}$ radio power. In the complete sample, we have $27 \mathrm{BCGs}$, including cases like A400 (double BCG, 3C75, Fig. 1) and clusters with a clear double structure (e.g. A1314) where we observed the BCG of both substructures.

New observations were obtained at the VLBA at $6 \mathrm{~cm}$ in phase referencing mode. Each source was observed for about $3 \mathrm{hrs}$ to assure a good uv-coverage and a low noise level. The resolution of 
Table 1: Results for the expanded sample: in the first column, we report the Abell cluster hosting the BCGs of our sample, in column 2, we put Y if the Abell cluster shows a cool core, N if it doesn't, SCF for small cooling flow and MCF is for middle cooling flow, in column 3 there are the names of BCGs. Column 4 refers to notes where (1) indicates clusters where we observed the BCG with VLBA observations at 6 $\mathrm{cm}$, (2) means that the BCG is a well known radio galaxy with published VLBI data, (3) is for well known BCG outside our complete sample with mas observations. Column 5 is for the large scale morphology of the BCG: we use WAT for Wide Angle Tail radiosource, HT for Head Tail radiosource, MSO for medium symmetric source. In the last column, we mark the parsec scale structure: one sided, two sided or n.d. for the non detections.

\begin{tabular}{|c|c|c|c|c|c|}
\hline Abell Cluster & cool core & BCG & notes & Large scale & VLBI \\
\hline \multirow[t]{2}{*}{ A400 } & $\mathrm{N}$ & $3 \mathrm{C} 75 \mathrm{~A}$ & 1 & WAT & one sided \\
\hline & $\mathrm{N}$ & $3 \mathrm{C} 75 \mathrm{~B}$ & 1 & WAT & one sided \\
\hline A407 & $\mathrm{N}$ & UGC2489 & 1 & Tail rs & one sided \\
\hline A539 & $\mathrm{N}$ & UGC3274 & 1 & radio quiet & n.d. \\
\hline A569 & $\mathrm{N}$ & NGC2329 & 1 & WAT & one sided \\
\hline A576 & $\mathrm{N}$ & CGCG261-059 & 1 & Tail rs & one sided \\
\hline A690 & $\mathrm{N}$ & B2 0836+29 II & 3 & WAT & one sided \\
\hline A779 & $\mathrm{N}$ & NGC2832 & 1 & radio quiet & n.d. \\
\hline A1185 & $\mathrm{N}$ & NGC3550 & 1 & radio quiet & n.d. \\
\hline A1213 & $\mathrm{N}$ & 4C29.41 & 1 & FRI & one sided \\
\hline A1228 & $\mathrm{N}$ & IC2738 & 1 & radio quiet & n.d. \\
\hline \multirow[t]{2}{*}{ A1314 } & $\mathrm{N}$ & IC708 & 1 & WAT & one sided \\
\hline & $\mathrm{N}$ & IC712 & 1 & small WAT & n.d. \\
\hline \multirow[t]{2}{*}{ A1367 } & $\mathrm{N}$ & NGC3842 & 1 & small WAT & n.d. \\
\hline & $\mathrm{N}$ & 3C264 & 2 & HT & one sided \\
\hline A1656 & $\mathrm{N}$ & NGC4874 & 1 & small WAT & one sided \\
\hline A2147 & $\mathrm{N}$ & UGC10143 & 1 & small WAT & n.d. \\
\hline \multirow[t]{2}{*}{ A2151 } & $\mathrm{N}$ & NGC6041 & 1 & small WAT & core \\
\hline & $\mathrm{N}$ & NGC6047 & 1 & compact core+symmetric jets & n.d. \\
\hline A2162 & $\mathrm{N}$ & NGC6086 & 1 & FRI, relic source & n.d \\
\hline A2197 & $\mathrm{N}$ & NGC6173 & 1 & point source & one sided \\
\hline A2634 & $\mathrm{N}$ & $3 \mathrm{C} 465$ & 2 & WAT & one sided \\
\hline A2666 & $\mathrm{N}$ & NGC7768 & 1 & Tail rs & one sided \\
\hline A262 & $\mathrm{Y}$ & NGC708 & 1 & double-no core,jets & core \\
\hline A347 & SCF & NGC910 & 1 & radio quiet & n.d. \\
\hline A426 & $\mathrm{Y}$ & $3 \mathrm{C} 84$ & 2 & Compact core + Halo & two sided \\
\hline A780 & $\mathrm{Y}$ & Hydra A & 3 & double & two sided \\
\hline A1795 & $\mathrm{Y}$ & $4 \mathrm{C} 26.42$ & 3 & double & two sided \\
\hline A2052 & $\mathrm{Y}$ & $3 \mathrm{C} 317$ & 3 & bright core+halo (FRI) & two sided \\
\hline A 2152 & $\mathrm{MCF}$ & UGC10187 & 1 & Tail rs & n.d. \\
\hline A2199 & $\mathrm{Y}$ & 3C 338 & 2 & double restarted & two sided \\
\hline A2390 & $\mathrm{Y}$ & B2151+174 & 3 & MSO & two sided \\
\hline A 2597 & Y & PKS 2322-123 & 3 & asymmetric radiosource (FRI) & two sided \\
\hline
\end{tabular}




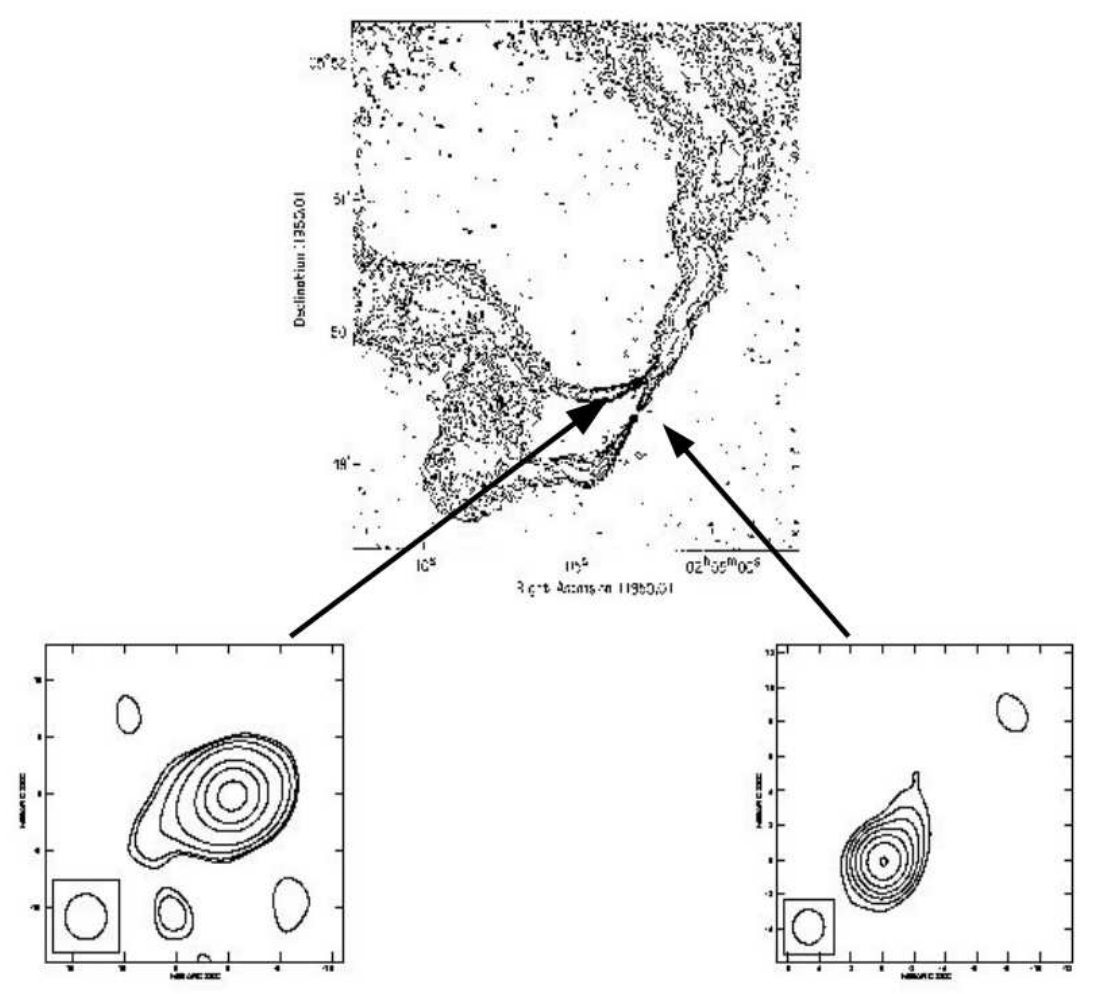

Figure 1: In the center: VLA image at $6 \mathrm{~cm}$ of 3C 75A and 3C 75B [5], the double BCG in the non cool core cluster A400. Contour intervals are $(-1,1,2,4,8,16,32,64,128,256,512) \times 0.1 \mathrm{mJy} / \mathrm{beam}$. The HPBW is $1.4 \times 1.4$ arcsec with P.A. $=0^{\circ}$. On the right: one-sided VLBI image [8] of 3C75A. Contours levels are $0.3,0.6,1.2,2.4,4.8,9.6,19.2$ mJy per clean beam and the HPBW is $2 \times 2$ mas with P.A. $=0^{\circ}$. On the left: one-sided VLBI image [8] of 3C75B. Contours levels are $-0.3,0.3,0.4,0.8,1.8,7,14,28 \mathrm{mJy} / \mathrm{beam}$ and the HPBW is $4 \times 4$ mas with P.A. $=0^{\circ}$.

the final maps is typically $3 \times 1.8$ mas and the noise level is $\sim 0.1 \mathrm{mJy} / \mathrm{beam}$. The detection rate in the complete sample (literature and new data) is $\sim 60 \%$. Obtained results are shown in Tab. 1 together with literature data (expanded sample, see next). A comparison between BCGs in cooling and non-cooling clusters suggests a difference in the properties of the parsec scale structures, but numbers are too small to properly discuss it.

To improve our statistics, we performed a search in the literature and archive data looking for VLBI data of BCGs in Abell clusters with DC $>2$. We added to our complete sample the following clusters: A690, A780, A1795, A2052, A2390, and A2597 (expanded sample). Results are presented in Table 1. In the expanded sample, we find a remarkable dominance of two-sided sources in relaxed clusters $(70 \%)$, and of one-sided (56\%) or non-detected (39\%) sources in merging systems, as shown in Tab.2.

The difference between relaxed and non relaxed clusters is evident. The presence of one-sided jets could suggest relativistic Doppler boosting. In this hypothesis two-sided jet structures would represent sub-relativistic velocities. However the jet properties can also be related to different 
Table 2: BCG counts in the complete (nearby) sample and expanded one. We report the number of BCG according to the cluster morphology and pc scale morphology. Note that most of undetected sources in VLBA observations are in BCG that are radio quiet (or faint) also in VLA observations.

\begin{tabular}{ccccccc}
\hline \hline Sample & $\begin{array}{c}\text { Cluster } \\
\text { morphology }\end{array}$ & Number & two-sided & one-sided & point & N.D. \\
\hline Complete & cool core & 5 & $2(40 \%)$ & - & 1 & 2 \\
& non cool core & 22 & - & $12(55 \%)$ & 1 & 9 \\
\hline Expanded & cool core & 10 & $7(70 \%)$ & - & 1 & 2 \\
& non cool core & 23 & - & $13(56 \%)$ & 1 & 9 \\
\hline \hline
\end{tabular}

properties in jet formation or to the interaction with a different ISM. In BCG at the center of cooling clusters the gas density in the ISM region is expected to be higher. Therefore we can assume a strong interaction of the jet at parsec resolution with the environment. This difference
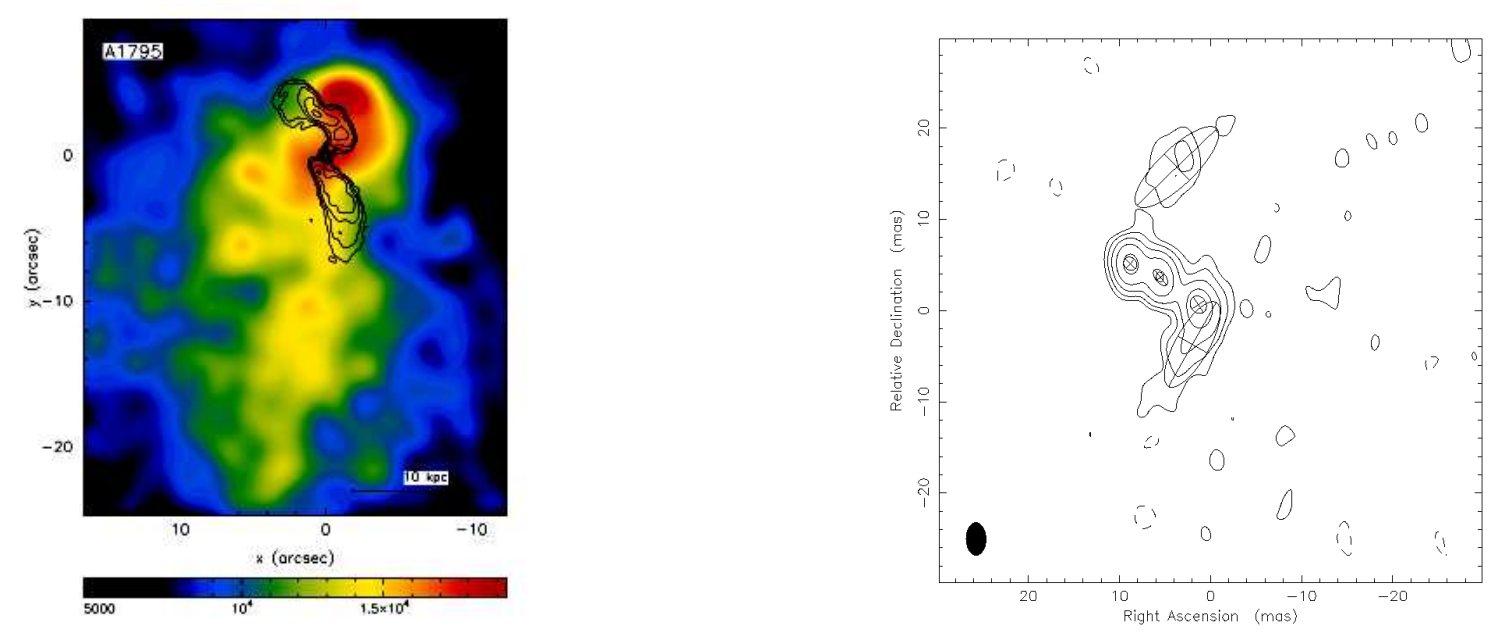

Figure 2: On the left: an overlay [6] of the $3.6 \mathrm{~cm}$ radio emission of 4C 26.42 (BCG of Abell 1795) at 0.37 -arcsec resolution on the Chandra X-ray image convolved with a 2-arcsec Gaussian. The radio contours start at $0.08 \mathrm{mJy} / \mathrm{beam}$ and increase by factors of 2 to just below the peak in the image of $33 \mathrm{mJy} / \mathrm{beam}$. On the right: two-sided VLBI image at $5 \mathrm{GHz}$ with components given by Modelfit. Contours of VLBI image are $-0.4,0.4,0.8,1.6,3.2,6.4 \mathrm{mJy} / \mathrm{beam}$ and the $\mathrm{HPBW}$ is $3.6 \times 2.2$ mas with P.A. $=0.543^{\circ}$.

of radio morphology between BCGs in cool core and non cool core clusters could be also related to a different availability of gas in the nuclear regions. This suggestion is supported by literature data on BCGs of more distant clusters as e.g. 4C26.42 (Fig.2), the BCG in the cool core cluster A1795, where VLBI images show a distorted symmetric structure [8], and Hydra A (the BCG of the cool core cluster A780), where Taylor (1996) [9] suggested that the emission from the symmetric parsec scale jets is more dependent on interactions with the surrounding material than on Doppler boosting.

On the other hand, the one-sided structure in non cool core clusters might due to Doppler boosting effects in relativistic, intrinsically symmetric jets. This is the case for example of $3 \mathrm{C} 75$ 
A and B (Fig.1), 3C465 [10] and more.

We conclude that the possibile dichotomy between BCGs in cool core and non cool core clusters could be due not to intrinsic jet differences but to different ISM conditions. We plan to observe a larger sample of BGCs in cooling and relaxed clusters with VLBA to improve our statistic.

\section{Acknowledgments.}

We thank the organizers of a very interesting meeting. The National Radio Astronomy Observatory is operated by Associeted Universities, Inc., under cooperative agreement with the National Science Foundation.

\section{References}

[1] Best P.N., Kaiser C. R., Heckman T. M., Kauffmann G. , 2006 MNRAS 368, 67

[2] Birzan L. et al. 2008 ApJ in press (astro-ph 0806.1929)

[3] Dunn, R.J.H. and Fabian, A.C. 2006 MNRAS 373, 959

[4] Gentile G., Rodriguez C., Taylor G. B., Giovannini G., Allen S. W., Lane W. M., Kassim N. E., 2007, ApJ 659, 225

[5] Eilek, Jean A.; Owen, Frazer N., 2002, ApJ 567, Issue 1, pp. 202-220.

[6] Fabian A. C., Sanders J. S., Ettori S., Taylor G. B., Allen S. W., Crawford C. S., Iwasawa K., Johnstone R. M., 2001, MNRAS, Volume 321, Issue 2, pp. L33-L36.

[7] Gitti M., Ferrari C., Domainko W., Feretti L., Schindler S., 2007 A\&A 470, 25

[8] Liuzzo et al. in preparation

[9] Taylor G.B., 1996, ApJ 470, 394

[10] Venturi T., Castaldini C., Cotton W. D., Feretti L., Giovannini G., Lara L., Marcaide J. M., Wehrle A. E, 1995, ApJ 454, 735

[11] Yen-Ting Lin and Joseph J. Mohr, 2004 ApJ 617, 879 